\section{Psychosocial distress associated with disfiguring eye conditions}

\author{
A Clarke1, N Rumsey², JRO Collin ${ }^{3}$ and \\ M Wyn-Williams ${ }^{4}$
}

Eye (2003) 17, 35-40. doi:10.1038/

sj.eye. 6700234

Purpose of study Visible disfigurement is associated with a range of psychosocial difficulties; however, the problems encountered by patients with eye conditions are under-researched. This study aimed to establish the extent and type of psychosocial distress in this patient population, and to explore the extent to which patients' needs are currently met.

Method Standardised measures (anxiety, depression, appearance-related distress, and quality of life) and a semistructured interview (exploring individual experiences and satisfaction with care) were administered to 153 consecutive attenders at outpatient eye clinics in three different hospitals. Responses were analysed using descriptive statistics, multiple regression, and cluster analyses. Results Between 10 and $49 \%$ of the patient sample scored unfavourably on standardised measures in comparison with normative values. Cluster analyses revealed that more distressed patients typically exhibited higher levels of anxiety, depression, social anxiety, self-consciousness, and social avoidance. Quality of life scores were also less favourable. Distressed patients felt less well informed, less involved in their treatment, and less well supported in the clinic setting. Variability within the sample was high; however, the effects of the cause and duration of the condition were not significantly related to distress. Clinic staff identified a variety of constraints to meeting patients' need, including patient numbers, the lack of appropriate training, and the lack of referral possibilities. Conclusions A considerable proportion of patients with disfiguring eye conditions experience high levels of psychosocial distress. Several options exist for ways in which patients' needs might more effectively be met within the constraints currently facing care providers.
Keywords: disfigurement; psychosocial need; psychological distress; outpatients; patient satisfaction

\section{Introduction}

Visible disfigurement is a social disability, which has been described by McGrouther ${ }^{1}$ as the 'last bastion of discrimination in this country' (ie the UK). There is general acceptance that visible disfigurement is associated with a range of psychosocial difficulties; ${ }^{2}$ however, current care provision within the NHS focuses on medical and surgical interventions to treat appearance-related concerns.

Despite an increased understanding of the difficulties faced by people with a range of disfiguring conditions, the problems encountered by patients with eye conditions are under-researched. The impact of an injury or disease that affects the eye, even where quality of life measures have been employed, has been studied almost exclusively in terms of the effects on vision (see, for example, Ref. 3). Very little is known about the psychological impact of an unusual appearance in visible eye conditions, which is surprising, given the central role of the eyes in both communication and perceived physical attractiveness. Frewin et $a l^{4}$ have suggested that psychological issues related to appearance are particularly important in thyroid eye disease and this finding is supported by clinical observation; however, their conclusion that the extent or severity of exophthalmos is predictive of psychological distress is an unusual finding in the disfigurement literature. Recent research has consistently indicated that, instead, psychosocial factors such as high levels of selfesteem, effective social skills, and a good quality social support network are powerful mediators of problems associated with visible disfigurement. ${ }^{5}$

\begin{abstract}
${ }^{1}$ Adult Services Changing Faces London, UK
\end{abstract}

${ }^{2}$ Centre for Appearance \& Disfigurement Research University of the West of England

Bristol, UK

${ }^{3}$ Moorfields Eye Hospital London, UK

${ }^{4}$ UWE, Bristol, UK

Correspondence: Dr Nichola Rumsey Centre for Appearance \& Disfigurement Research Department of Psychology University of the West of England

Fishponds, Bristol BS16 2JP UK

Tel/Fax: +44 1173444356 E-mail: Nichola.Rumsey@ uwe.ac.uk

Received: 7 December 2001 Accepted in revised form: 11 April 2002 
As part of a larger project identifying the needs of outpatients with a large range of disfiguring conditions, information was collected from three clinics treating patients with a range of disfiguring eye conditions. The aims of the project were to establish the extent and type of psychosocial distress in outpatients with eye conditions and to explore the extent to which these needs are met by current care provision.

\section{Materials and methods}

Ethical approval for the study was given by the appropriate LRECs. Consecutive outpatient attenders from three different outpatient eye clinics were approached over a 6-month period. Potential participants aged 18 years or more with a disfiguring eye condition were invited to read an information sheet. Those who were unable to read the sheet without help (either as a result of language or learning difficulties) were excluded from the study on the grounds that they would have been unable to complete the standardised scales.

Participants were also screened for mental disorder using a combination of behavioural observation and questions relating to any previous psychiatric history.

The nature of the study was further explained by the interviewer. Participants read and signed a proforma (the wording of which had been previously approved by the relevant ethical committee) to indicate their consent to the study. Sample characteristics are shown in Table 1.

Table 1 Sample characteristics

\begin{tabular}{lccccc}
\hline & $\begin{array}{c}\text { Participant } \\
\text { numbers }\end{array}$ & $\begin{array}{c}\text { Males } \\
(\%)\end{array}$ & $\begin{array}{c}\text { females } \\
(\%)\end{array}$ & $\begin{array}{c}\text { Mean age } \\
\text { (years) }\end{array}$ & $\begin{array}{c}\text { Age } \\
\text { range }\end{array}$ \\
\hline Clinic 1 & 50 & 44 & 56 & 55 & $19-83$ \\
Clinic 2 & 30 & 50 & 50 & 50 & $19-79$ \\
Clinic 3 & 73 & 40 & 60 & 49 & $18-79$ \\
Total & 153 & & & & \\
\hline
\end{tabular}

Participants were asked to complete a series of standardised measures and a semistructured interview. The standardised measures were chosen (1) on the basis of a review of the literature to identify the psychological factors most commonly implicated in appearance-related distress, ${ }^{6}(2)$ in relation to the suitability of the proposed measures to assess the extent and type of psychosocial need in people with disfiguring conditions, ${ }^{7}$ and (3) for their ease of completion in the clinic setting. These measures comprised the following:

- Hospital Anxiety \& Depression Scale (HADS); ${ }^{8}$

- Derriford Appearance Scale-Short Form ${ }^{9}$ measuring appearance-related concerns and social avoidance; and
- World Health Organisation Quality of Life Scale-Short Form (WHOQoL Bref). ${ }^{10}$

The semistructured interview explored individual concerns, strengths and difficulties in social functioning, coping strategies, and levels of perceived support. Respondents were also asked a series of questions relating to their experience at the clinic.

Quantitative data were analysed using descriptive statistics, analyses of variance, correlations, and cluster analyses. Open-ended responses to interview questions were explored using content analysis.

\section{Results}

Table 2 illustrates the presenting conditions in each clinic, with Table 3 indicating the duration for which patients had experienced the condition.

Table 2 Cause of the condition (\%)

\begin{tabular}{lcccc}
\hline & Congenital & Trauma & Disease & Missing/other \\
\hline Clinic 1 & 8 & 40 & 50 & 2 \\
Clinic 2 & 13 & 13 & 71 & 3 \\
Clinic 3 & 19 & 26 & 50 & 5 \\
\hline
\end{tabular}

Table 3 Duration of condition (\%)

\begin{tabular}{lcccccc}
\hline & $\begin{array}{c}<6 \\
\text { months }\end{array}$ & $\begin{array}{c}6 \text { months }-1 \\
\text { year }\end{array}$ & $\begin{array}{c}1-2 \\
\text { years }\end{array}$ & $\begin{array}{c}2-5 \\
\text { years }\end{array}$ & $\begin{array}{r}5-10 \\
\text { years }\end{array}$ & $\begin{array}{c}10+ \\
\text { years }\end{array}$ \\
\hline Clinic 1 & 12 & 2 & 0 & 12 & 4 & 70 \\
Clinic 2 & 3 & 9 & 3 & 37 & 9 & 39 \\
Clinic 3 & 5 & 11 & 5 & 22 & 11 & 44 \\
\hline
\end{tabular}

\section{Anxiety and depression}

The results for anxiety and depression are presented in Table 4. Interpretation of the HADS scores was based on the following categories relating to clinical 'case' levels of distress derived from Zigmond and Snaith: ${ }^{8}$

$\begin{array}{ll}7 \text { or less } & \text { Noncase } \\ 8-10 & \text { Borderline case } \\ 11+ & \text { Definite case }\end{array}$

Cumulative frequencies indicated that $10 \%$ of participants in Clinic 1 exhibited 'case' levels of anxiety. The figures for Clinics 2 and 3 were considerably higher, at 45 and $34 \%$. Although mean levels of depression were low, cumulative frequencies indicated that $3 \%$ of participants from Clinic 1, 14\% from Clinic 2, and 18\% from Clinic 3 exhibited 'case' levels of depression.

\section{Social anxiety and social avoidance}

Scores on the Derriford Short Form range between 0 and 85. The higher the score, the more anxious a person is 
Table 4 HAD scores for anxiety and depression

\begin{tabular}{lclll}
\hline & Mean & SD & Min & Max \\
\hline HAD anxiety & & & & \\
Clinic 1 & 6.5 & 3.66 & 0 & 17 \\
Clinic 2 & 10.22 & 4.85 & 1 & 20 \\
Clinic 3 & 8.2 & 4.9 & 0 & 19 \\
Total project & 8.6 & 4.1 & 0 & 24 \\
HAD depression & & & & \\
Clinic 1 & 4.6 & 3.4 & 0 & 14 \\
Clinic 2 & 6.18 & 4.10 & 0 & 14 \\
Clinic 3 & 5.6 & 4.4 & 0 & 17 \\
Total project & 5.8 & 3.4 & 0 & 18 \\
\hline
\end{tabular}

Table 5 Derriford Short Form scores

\begin{tabular}{lcrcc}
\hline & Mean & SD & Min score & Max score \\
\hline Clinic 1 & 36.8 & 9.5 & 18 & 58 \\
Clinic 2 & 46.7 & 10.3 & 27 & 64 \\
Clinic 3 & 44.7 & 14.5 & 24 & 78 \\
Total project & 40.2 & 10.4 & 17 & 80 \\
\hline
\end{tabular}

about his/her appearance. A value of $47(\mathrm{SD}=11)$ was found by Moss $^{9}$ to represent the mean value for people seeking plastic surgery for appearance-related concerns. Although the mean values for this sample were below this level, the range (see Table 5) and cumulative frequencies of those with scores greater than 47 (16\% for Clinic 1, 45\% for Clinic 2, and 34\% for Clinic 3) indicated that many participants experienced considerable levels of appearance-related distress and social avoidance.

Quality of life The WHOQoL Bref has four domainsphysical, psychological, social, and environmental. A score of 15 is described by the WHO as the normative mean score on all domains, with higher scores denoting better quality of life. Mean scores (physical domain 16.85, psychological domain 15.07, social domain 16.3, environmental domain 17.1) suggested that participants reported reasonably good quality of life scores; however, the standard deviations and ranges were large indicating considerable variability within the sample. Cumulative frequencies revealed that $21-72 \%$ of participants scored below normative levels, with particularly adverse scores on the psychological domain.

Correlations showed no significant relationships between psychological distress and either age or the length of time for which patients had experienced the condition. Analyses of variance showed no significant differences in the scores on the standardised measures relating to gender or to the cause of the condition.
Interview findings Participants were asked To what extent has your appearance affected your lifestyle? Using a scale from 0 (not at all) to 100 (very much), the mean value of 55 was unremarkable; however, the large standard deviation (32.4) and range of scores (0-100) indicated that many felt their appearance was having a considerable effect. In response to the question How noticeable do you feel your condition is to other people, a similar pattern emerged, with a mean score of 61.6, a standard deviation of 29.9, and scores ranging from 0 to 100 . These scores compared unfavourably with the overall project mean of 55.5 .

When asked Do you worry about the appearance of your eye condition, participants once again responded on a scale of 0 (not at all) to 100 (very much). Table 6 illustrates the variation in responses between the three clinics, and the comparison with the overall project statistics. Once again, the large standard deviations and ranges indicated enormous variability in the sample, with a considerable proportion experiencing notable levels of distress.

Additional interview findings Participants were receiving variable levels of support in relation to their appearance. Those receiving better quality social support (assessed through self-reported ratings on visual analogue scales) did not feel that they were coping better than those feeling less well supported. Many expressed the desire for a designated professional within the clinic with whom they could discuss their difficulties. Participants reported a variety of difficulties associated with their condition. The most common included dealing with negative reactions from the general public, meeting new people, feelings of low self-confidence, and restrictions to social life due to anxieties about being seen in public. A variety of coping strategies were reported, including avoidance, distraction, cognitive restructuring, and seeking out social support.

Experience of clinic care Participants were asked a series of questions concerning their experience at the clinic. The responses, illustrated in Table 7 , showed considerable variation between clinics.

When asked whether they would like to be more involved in treatment decisions, $6 \%$ of participants in Clinic 1 said they would like to be more involved, with higher percentages in Clinic $2(24 \%)$ and Clinic $3(24 \%)$ expressing this desire. A total of $80 \%$ of participants in Clinic 1, 9\% of Clinic 2, and 20\% of Clinic 3 wanted more information about their treatment to date, with $8 \%$ (Clinic 1), 9\% (Clinic 2), and 12\% (Clinic 3) feeling they wanted to know more about future treatment. 
Table 6 Responses to the question 'Do you worry about the appearance of your eye condition?'

\begin{tabular}{llll}
\hline & Mean & \multicolumn{1}{c}{ SD } & Range \\
\hline Clinic 1 & 37.7 & 33.0 & $0-100$ \\
Clinic 2 & 63.7 & 26.33 & $2-100$ \\
Clinic 3 & 58.5 & 36 & $0-100$ \\
Overall project score & 49.74 & 31.8 & $0-100$ \\
\hline
\end{tabular}

Visual analogue scale, ranging from $0=$ not at all to $100=$ very much. Additional findings from the interview were included

In order to explore the patterns of associations between the measures, standard scale and visual analogue scale scores were subjected to cluster analyses. The resulting pattern of significant correlations $(P<0.05)$ revealed that higher levels of anxiety were associated with greater levels of social anxiety and social avoidance $(r=0.66)$, higher levels of worry about appearance $(r=0.49)$, the belief that the condition was more noticeable to other people $(r=0.52)$ and less favourable perceptions of social support $(r=-0.25)$. These patients also found treatment explanations offered in the clinic less easy to understand $(r=-0.32)$.

Higher levels of depression were associated with greater social anxiety and social avoidance $(r=0.57)$, higher levels of worry about appearance $(r=0.34)$ and with less favourable perceptions of social support $(r=-0.33)$. Patients with higher levels of depression also found it more difficult to ask questions about their condition in the clinic setting $(r=-0.24)$. The following illustrative quotes from the interviews summarise the distress experienced by many.

I don't have a close relationship with a man anymore... If I can't accept my new appearance, how can I expect anyone else to?

The condition has affected my whole lifestyle....I have had to temporarily sacrifice my career...it has affected my relationship with my partner and my children as some days I am a social recluse... I can get very depressed and tearful...I can't wait for this dreadful period to be over...

I feel that I have to get to know myself all over again who is this person I am looking at in the mirror?

People can be so cruel with their reactions....I don't know what is worse... when they make comments or when they just stare....they just don't know anything about the condition or what I am going through...

You just take your appearance for granted until something happens to it...

The researchers also met with clinic staff to elicit their views on the psychosocial needs of patients and the constraints and difficulties experienced when trying to address these needs. Staff in all three clinics felt that a substantial proportion of patients were presenting with psychosocial needs in addition to physical and functional concerns. Some admitted to nursing staff that they had extensive difficulties, whereas others were clearly reticent in discussing the psychological and social ramifications of their condition. Staff felt that several factors contributed to their inability to address patients' concerns. These included time pressures from patient numbers, a lack of sufficient privacy in the outpatient

Table 7 Self-reported experiences of clinic care

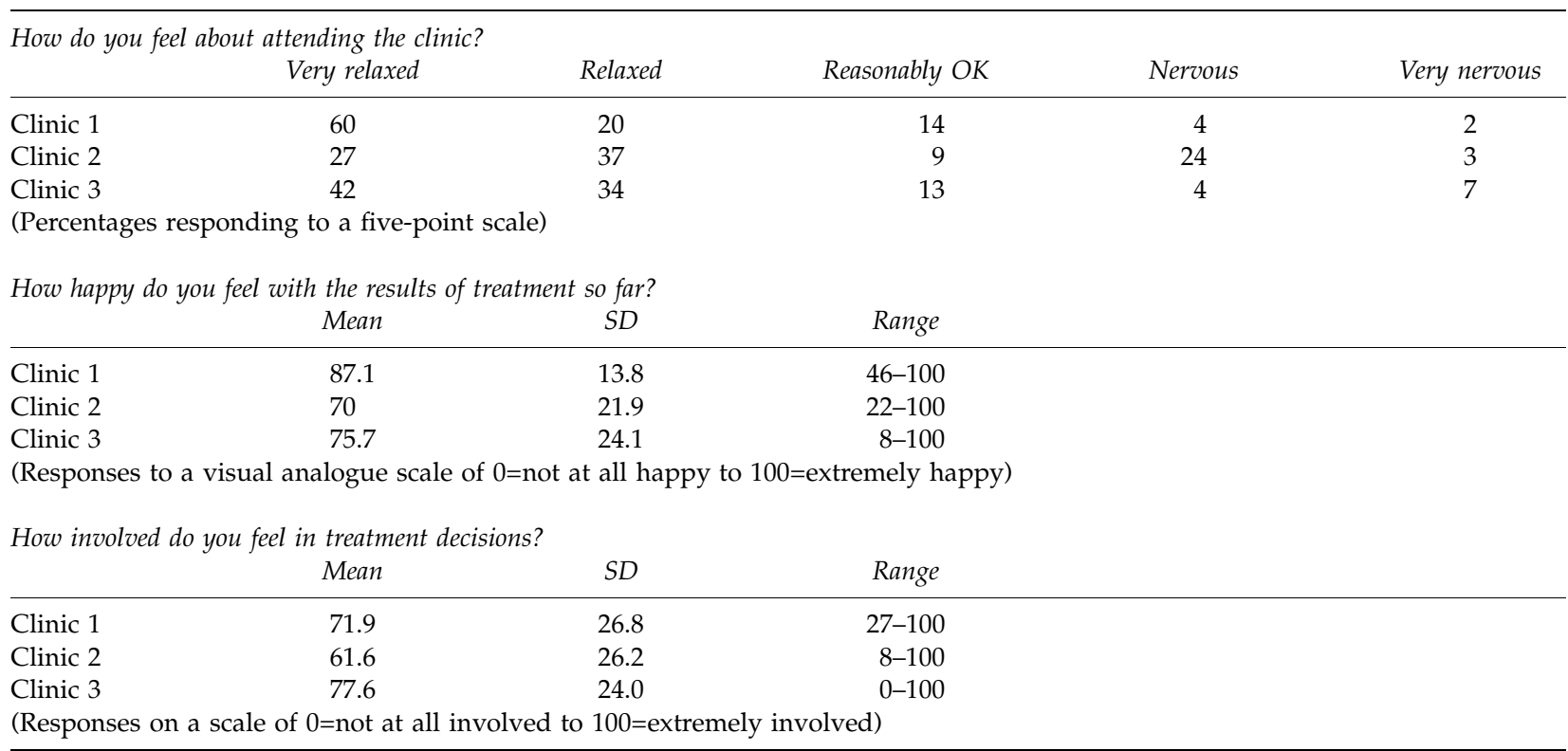


setting to facilitate the discussion of sensitive issues, a lack of specialist training, and the absence of sources for appropriate specialist referral. One clinic had a specialist nurse with a counselling qualification who was routinely contacted in the case of designated procedures, including exenteration and enucleation of the eye. However, no screening process was available to identify other patients experiencing psychosocial distress, and no protocol had been developed for supporting these patients.

\section{Discussion}

The results indicate that in addition to physical and functional difficulties, a substantial proportion of the outpatients were experiencing psychosocial difficulties associated with the appearance of their eye(s). The intensity and timing of these difficulties varied considerably within the group, and the results confirmed the findings of recent research relating to other visibly disfiguring conditions which indicate that psychosocial adjustment cannot be predicted from the physical characteristics of the condition (eg the aetiology or the duration of the condition).

The study suggests differences in the level of psychosocial distress according to the clinic attended (see Tables 4-6). Patients in Clinic 1 reported the most favourable levels of anxiety, depression, and social anxiety. Their ratings of the experience of care were the most positive for the majority of measures (see Table 7). The least favourable scores were produced by patients in Clinic 2, whereas indicators of variability within the sample were highest for Clinic 3. A large-scale study is necessary to clarify the many potential factors contributing to these differences between clinics.

Patients found it easier to report physical problems and practical concerns to the care team than to raise psychosocial issues. Many patients expressed the desire to talk about their problems should an appropriate professional be available in the clinic. In reporting the results to each clinic, the research team has recommended that clinic teams consider ways of making it possible for patients to raise and discuss their psychosocial concerns in a supportive atmosphere. Changes in the 'agenda' of the clinic could be facilitated through the provision of study days for staff designed to raise awareness of common problems and of ways of addressing difficulties experienced by patients.

The individual variation in levels of adjustment and distress suggests that a broad-brush approach to meeting patient need would be inappropriate and that an individualised approach to the assessment of need and the provision of support is required. This could be achieved in a number of ways including the development of a specialist nurse role, ${ }^{11}$ the establishment of appropriate referral routes for support and intervention, or through the inclusion of a psychosocial specialist as a member of the care team. The latter option has the benefit of addressing psychosocial issues as a matter of routine care and avoiding the potentially stigmatising nature of a referral for psychological support.

As a cross-sectional study, this research suffers from the usual problems associated with 'one-stop' data collection. However, the results reveal that a significant proportion of patients with visible eye conditions experience high levels of psychosocial distress and that these patients are less positive in their ratings of care provision. Many fail to seek help for their psychosocial concerns from clinic staff, and in the context of a busy clinic, the wider issues of psychosocial adjustment to disfiguring conditions can easily be overlooked. Patient needs could more effectively be met if appropriate psychological support were provided.

\section{Acknowledgements}

This work was carried out in the Out-Patient Departments of the Moorfields Eye Hospital, London; The Bristol Eye Hospital \& The Artificial Eye Centre, Bristol. The authors wish to acknowledge the help and support offered by staff at these centres, the contribution made to data collection by Veronica Kish, and the statistical expertise provided by Dr Paul White, Faculty of Computing, Statistics \& Mathematics, University of the West of England, Bristol, UK. This research was funded jointly by The Nuffield Trust \& The King's Fund.

\section{References}

1 McGrouther DA. Facial disfigurement: the last bastion of discrimination. BMJ 1997; 314: 991.

2 Lansdown R, Rumsey N, Bradbury E, Carr T, Partridge J (eds). Visibly Different: Coping with Disfigurement. Butterworth Heinemann: London, 1997.

3 Hart P, Chakravarthy U, Stevenson M. Questionnaire based survey on the importance of quality of life measures in Ophthalmic practice. Eye 1998; 12: 124-126.

4 Frewin SE, Dickinson AJ, Kendall Taylor P, Perros P. Thyroid eye disease. Newslett Thyroid Eye Dis Assoc 1999; 45: 8-11.

5 Moss TP. Individual differences in psychological adjustment to perceived abnormalities of appearance. Unpublished PhD thesis, University of Plymouth, 1997.

6 Clarke AC. Psychosocial aspects of facial disfigurement: problems management and the role of a lay-led organisation. Psychol Health Med 1999; 4(2): 127-142.

7 Carr T. Assessment and measurement in clinical practice. In: Lansdown R, Rumsey N, Bradbury E, Carr T, Partridge. J (eds). Visibly Different: Coping with Disfigurement. Butterworth Heinemann: London, 1997, pp 131-146. 
8 Zigmond AS, Snaith RP. The Hospital Anxiety \& Depression Scale. Acta Psychiatr Scand 1983; 67: 361-370.

9 Moss TP. Individual variation in adjusting to visible differences. In: Lansdown R, Rumsey N, Bradbury E, Carr T, Partridge J (eds). Visibly Different: Coping with Disfigurement. Butterworth Heinemann: London, 1997, pp 121-130.
10 The WHOQOL Group. Development of the World Health Organisation WHOQOL-Bref Quality of Life Assessment. Psychol Med 1998; 28: 551-558.

11 Clarke A, Cooper C. Psychosocial rehabilitation after disfiguring injury or disease; investigating the training needs of specialist nurses. J Adv Nurs 2001; 34(7): 18-26. 